\section{COMPARISON INTRA ARTICULAR STEROID VS. HYDRAULIC DISTENTION FOR THE TREATMENT OF FROZEN SHOULDER}

\section{Singh G.P}

Address for correspondence

$$
\text { Dr.G.P.Singh }
$$

Professor, Department of Orthopaedic

Surgery, U.C.M.S. Teaching Hospital,

Ranigaon, Bhairahawa, Nepal.

Email:-gp_singh1@hotmail.com

\section{ABSTRACT \\ Background}

Frozen shoulder is a chronic fibrosing condition of the capsule of the shoulder joint.

\section{OBJECTIVE}

The objective of the study was to compare the result of treatment of frozen shoulder by Hydraulic Distention under local anesthesia with steroid and intra articular steroid alone.

Sample Size: 60 patients were taken in this study and were divided into two groups.

Duration and Setting: The study was conducted in 2005-2006 treated in the department of orthopaedic, Janaki medical college Teaching Hospital, Janakpur.

\section{Materials and Methods:}

It was a prospective, observational, comparative and clinical study. Sixty patients were selected from Dhanusha District of Janakpur, Nepal. They were divided into two groups. Since January 2005 to January 2006 periods in the department of orthopedics surgery, Janaki Medical College \& Teaching Hospital, Janakpur. The Group I was treated by hydraulic distention of glenohumeral joint with $50 \mathrm{ml}$ normal saline with steroid, oral medicines (analgesics and muscle relaxants) and exercises. Patients in group II were treated by intra-articular steroid followed by oral medicines (analgesics and muscle relaxants) and exercises.

Results: The average age of patients in group I was $52 \pm 2.16$ years and $50 \pm$ 2.03 in group II. There were $37(63 \%)$ females and $23(37 \%)$ males in our study. Female to male ratio was 1.7:1. All patients in group noted immediate pain relief with excellent return of range of motion (ROM) and resumption of normal sleep. At the end of 45 days of follow up all patients had returned to their normal daily activities. Most of them got excellent ROM and pain relief. All patients in group II noted immediate pain relief and resumption of almost normal sleep. At the end of 45 days of follow up most of the patient presented with good relief of pain but could not return to most of their normal activities because of less improvement in ROM.

Conclusions: Hydraulic distension technique is quick, safe and gives early results, so it should be considered first for the treatment of frozen shoulder.

Key Words: frozen shoulder, Hydrologic Distention, Intra articular steroid. 


\section{INTRODUCTION}

Frozen shoulder is a chronic fibrosing condition of the capsule of the shoulder joint, ${ }^{1}$ The term frozen shoulder as first used by Codman 1932 who described the common features as a pain of gradual onset, which is felt near the insertion of the deltoid, inability to sleep on the affected side, painful restriction of elevation and external rotation and a normal radiological appearance. ${ }^{12} \mathrm{He}$ stated that most cases resolved in about two years without treatment. Mare recently, ZuCkerman and Cuomo defined frozen shoulder, or adhesive capsulitis, as a condition of uncertain etiology characterized by substantial restriction of both active and passive shoulder motion that occurs in the absence of a known intrinsic shoulder disorder. $^{3}$

The cause of frozen shoulder remains enigmatic. ${ }^{2}$ Naviasor (1945) suggested a chronic inflammatory condition Lundberg (1969) suggested that the primary pathology was fibrosis \& fibroplasias. ${ }^{2}$ Recently arthroscopic studies showed that the capsule was contracted, there ware no adhesions and that the maximal area of abnormality was at the rotator interval. ${ }^{1}$ Frozen shoulder has been divided into three stages depending on its symptoms.

1. Freezing phase also called the painful phase. This condition lasts for 2-9 months.

2. Frozen phase also called the stiffening phase, lasts for 4-12 months.

3. Thawing phase also called the recovery phase, may lasts for 6-9 months. ${ }^{4,5}$

Adhesive capsulitis usually occurs during the fourth to sixth decades, affecting women more often than men. ${ }^{4}$ Bilateral involvement occurs in $10-40 \%$ of cases but frequently involves in the nondominant extremity. ${ }^{5}$ There is higher than normal association between frozen shoulder and diabetes mellitus. The incidence in general population is $2-5 \%$ while among diabetics is $10-20 \%{ }^{6}$

A variety of different treatments have been recommended and numerous studies have demonstrated successful results. The types of treatment have included benign neglect, chiropractic manipulation, oral corticosteroids, injection of corticosteroids, physical therapy exercises and modalities, manipulation under anesthesia and arthroscopic and open release of the contracture. $^{3}$ This study was conducted to evaluate and compare the results of treatment of frozen shoulder by hydraulic distension under local anesthesia with steroid and treatment with intra articular steroid therapy alone. A rapid, immediate result and cost effectiveness of hydraulic distension technique is also (to be) evaluated is this study.

\section{OBJECTIVES}

The purpose of study was to evaluate the results of treatment by hydraulic distension under local anesthesia with steroid and intra-articular steroid alone and to compare the result of two methods and to ascertain the range of motion gained, to assess patient compliance, subjective satisfaction, and to note any complication during follow up period in each group.

\section{MATERIALS AND METHODS}

Study Design: It was a prospective, observational, comparative, and clinical study.

Samples size: 60 patients were taken in this study and were divided into two groups.

Duration and Setting: The study was conducted in 2005-2006 treated in the department of Orthopaedic, Janaki Medical College, Janakpur.

Methodology: The patients were divided into two groups. Group I were treated by 
hydraulic distension of glenohumeral joint with $50 \mathrm{ml}$ normal saline under local anesthesia with steroid followed by oral medicines (analgesics and muscle relaxants) and exercises. Patients in group II were treated by intra-articular steroid followed by oral medicines (oral analgesics and muscle relaxants) and exercises.

\section{PROCEDURE}

Detailed history was taken and physical examination was done. X-rays of the shoulder joint AP view were obtained. Unbiased randomization of cases was taken in each group. The patients in group-I were managed initially by preparing skin with povidone iodine solution. $3 \mathrm{ml}$ of $1 \%$ plain lidocaine was injected into the skin and soft tissues overlying the joint capsule with a 23 gauge $\times 1$ " needle. There after, $3 \mathrm{ml}$ of $1 \%$ lidocaine was injected intraarticularly by anterior approach (with the needle passing through the deltopectoral groove and then below and medial to the tip of the coracoid process, through the coracobrachialis - biceps origin and subscapularis $^{2}$ with a 21 gauge $\times 1.5^{\prime \prime}$ needle), followed by steroid (40 mg triamcinolone acetonide mixed with $2 \mathrm{ml}$ of $1 \%$ lidocaine). Distension of the capsule was then performed with $50 \mathrm{ml}$ sterile saline solution. The patient then had active and assisted range of motion exercises under supervision. These consisted of pendulum exercises, abduction exercises along with oral medicines (NSAIDS, analgesics and muscle relaxants). The patients continued with regular home physical therapy exercises performed four times daily, assisted by the family, the patients in group-II were managed after preparing skin with povidone iodine solutions, with intra-articular steroid injection $(40 \mathrm{mg}$ triamcinolone acetoriide mixed with $3 \mathrm{ml}$ of $11 \%$ plain lido-caine). The injection was carried out through anterior approach as in group-I followed by exercises and medicines as in group-I.

Follow up examination was done in each group on day $0,7,15,30$ and 45 . When range of motion exercises, pain grading, patient's satisfaction, complications and activity level was taken note of by the same observer (author) on each visit to decrease interobserver bias. Functional assessment of the shoulder was done by scoring system modified from constant score by constant CR an Murley AHG, which is based on the obtained were compared between each group and with those of other published series.

\section{RESULTS}

The average age of patients in group I was $52 \pm 2.16$ years and $50 \pm 2.03$ in group II. There were 37 (63\%) females and $23(37 \%)$ males in our study. Female to male ratio was $1.7: 1$. All patients in group-I noted immediate pain relief with excellent return of range of motion (ROM) and resumption of normal sleep. At the end of 45 days of follow up all patients had returned to their normal daily activities Most of them got excellent ROM and pain relief. All patients in group-II noted immediate pain relief and resumption of almost normal sleep. At the end of 45 days of follow up most the patients presented with good relief of pain but could not return to most of their normal activities because of less improvement in ROM. At the end of 45 days $25(86 \%)$ patients got complete resolution of pain while $4(14 \%)$ patients complained of occasional mild pain in group-I.

Most of the patients treated by distention technique told that they had enjoyed first good night's sleep that they could not since the onset of their symptoms. Moreover consumption of oral analgesics and NSAIDS was markedly reduced earlier in group-I than in groupII. In group-II 18 (60\%) patients got complete resolution of pain while 12 
(40\%), patients complained of occasional pain of extreme of motion. There was no significant difference in pain relief as a whole $97 \%$ improvement in group-I and $92 \%$ improvement in group-II ( $P$ values>0.05).

Most of the patients in our study complained of extreme difficulty in toileting, personal hygiene problem, combing hair, change of dresses. This was due to restricted ROM and pain. Most of the patients in group-I after hydraulic distension technique got immediate freedom from above mentioned problems and this technique was much appreciated. Many patents called it as a miracle for them. We also observed marked elevation of mood in most of our patients in group-I In group-I $23(79 \%)$ patients resumed to their near full daily activities. Six (23\%) patients were not fully satisfied. In group-II most of the patients were not as happy as in group-I. only three $(10 \%)$ patients returned to near full daily activities. Twenty-two (73\%) patients could achieve half of their daily activities and were not satisfied as in group-I this all was due to less improvement in ROM.

Statistical analysis showed P-value> 0.001.

Average improvement in daily living was $91 \%$ in group-I and $78.5 \%$ in group-II

(P-value< 0.001).

In group-I we noted immediate remarkable improvement in ROM 46\%95\% (average 66\%) right after distension technique. Immediate improvement was as in sequence.

- Forward elevation $146^{\circ}(81 \%)$.

- Lateral elevation (abduction) $130^{\circ}(72 \%)$.

- External rotation $64 \%$.

- Internal rotation $47 \%$.

We did not find any immediate improvement in group-II. This marked difference continued till $45^{\text {th }}$ day. At the end of the $45^{\text {th }}$ day there was marked difference in improvement in ROM 84\% in group-I and $63 \%$ in group-II This difference was much significant that patients in group-I got excellent freedom of ROM and achieved nearly full daily work. While it was not as in group-II. ( $P$ value $<0.001)$. it is interesting to see that there was maximum improvement in forward elevation and lateral elevation (abduction). 93\% and $87 \%$ in group-I and $78 \%$ and $59 \%$ in group-II, respectively, and least improvement in external rotation and internal rotation, $83 \%$ and $73 \%$ and group-I, $63 \%$ and $53 \%$ in group-II, respectively.

As a whole we got excellent results $88 \%$ in group-I and fair to good $70 \%$ in group-II. Patients in group-I being treated by hydraulic distension technique were extremely happy.

\section{DISCUSSION}

The literature on frozen shoulder is replete with confusing and contradictory information concerning its etiology, pathology and treatment. $^{9}$ Steroid therapy has been advocated in frozen shoulder with the belief that inflammation has played an important role in the pathogenesis.

Naviaser found mild subsynovial inflammation in some cases, with edema, vascular dilatation, and mononuclear cell infiltration. However, Lundberg did not find significant inflammation in a study in which surgical specimens from patients with frozen shoulder and controls were interpreted blindly. Unfortunately, patients from whom surgical specimens are obtained may not demonstrate the early pathologic changes.

Corticosteroid therapy was suggested by Crisp and Kendall. Since then there have been many reports of uncontrolled experience with local corticosteroid. The reported results cover the entire gamut from no benefit to dramatic improvement. ${ }^{10}$

We also noted excellent response in one patient being treated by intra- 
articular steroid in group-II. In this study we evaluated the subjective and objective outcomes of the treatment of frozen shoulder with hydraulic distension under local anesthesia with steroid, compared with steroid alone. We found significant improvement in the ROM and analgesic usage in the group-I treated with hydraulic distension of $\mathrm{GH}$ joint under local anesthesia with steroid compared with steroid alone.

Frozen shoulder is a very protracted condition, which only resolves after years rather than months ${ }^{2}$. Distension treatment has been advocated as giving immediate pain reduction but without confirmation in the literature ${ }^{8}$. We found it true in our study.

Review of the previous literature on frozen shoulder demonstrates controversy about which of the many available treatment is best ${ }^{3}$. A number of different treatments, such as rest and anlgesicas, NSAIDS, local or oral steroids, physiotherapy, distension of the joint capsule, manipulation or a combination of these have been advocated. No standard treatment regime is universally accepted ${ }^{4}$. Recently arhro-scopic treatment and surgical release has been recommended for this condition. ${ }^{11}$

Some studies have found that local steroid injection gave some pain relief but without restoring movements and with no superior effect on the duration of symptoms compared with other treatments e.g. heat, physiotherapy, ice, local analgesic injections, manipulation or no treatment. Other studies have reported that local steroids were without advantage compared to physiotherapy or oral nonsteroidal anti- inflammatory. Manipulation of the shoulder under general anesthesia with an intra-articular steroid and local anesthesia injection has been recommended for frozen shoulder. This requires a more costly inpatient stay with general anesthesia and immediate post operative physiotherapy. There are also risks of fracture of the humeral neck and rupture of the rotator cuff when the procedure is performed by an inexperienced surgeon ${ }^{12}$.

Sharma RK, treated 32 patients, who had frozen shoulder which had not improved with physiotherapy (were treated) by manipulation under general anaestesia or by steroid injection and hydraulic distension under local anesthesia and recommended distension technique as it was easy to carry out and gave better results then manipulation ${ }^{14}$.

In a prospective study Van Royen said that hydraulic distension technique and manipulation under local anesthesia is safe, reliable and effective treatment for frozen shoulder ${ }^{15}$. Fareed and Gallivan treated 20 cases of frozen shoulder syndrome by hydraulic distension under local anesthesia. All these patients noted immediate resolution of previous pain and resumption of normal. ${ }^{11}$ Our patients also noted immediate good relief of pain and resumption of normal sleep in Group-I. Fareed and Gallivan added that local anesthesia with hydraulic distension is a safe, direct procedure that results in immediate relief of pain improved ROM, normal sleep, and complete resolution of the condition within a period of four weeks.

Fareed and Gallivan noted that the local anesthetic, along with hydraulic distension, lyses adhesions, and allows progressive motion. Virtually all patients with frozen shoulder complain of pain, sleep loss, and limited shoulder motion. Elimination of pain and restoration of a normal night sleep are immediately noted. Patients are pleased when their motion improves and they are able to regain normal function. It is impressive to see the change in mood and affect associated with the return to normal status. We also observed same feelings, or rather more than that in our patients, treated by hydraulic distension 
technique. Many patients called this technique a miracle. ${ }^{11}$

We also observed excellent ROM $(80-95 \%)$ in two patients and good ROM $(72-77 \%)$, in eleven patients treated by hydraulic distension techniques immediately after the procedure and in two patients ROM (62-69\%) was not good even after 45 days of follow up ( in group of distension technique). In a study Loyd JA an HM, and noted that the steroid injections have not been shown to improve the rate of return of shoulder motion $^{6}$.

In our study patients in group-II did not show significant improvement in ROM (average 63\%) after 45 days while in group-I improvement in ROM was excellent (average 84\%).

Pain relief was almost equal in both groups, $97 \%$ in group-I and $92 \%$ in group-II. We also observed immediate improvement in ROM (66\%), in group-I while it was not in group-II

We did not find any side effect other than mild pain during hydraulic distension technique in group-I except in one female patient who went into transient shock (vasovagal) during hydraulic distension technique but recovered immediately by conservative measure elevation both lower limbs, later on patient gave history of frightening of injection. So one should go for full cooperation and get confidence of the patient about hydraulic distension technique. Further studies are required to know more about etiology pathology and treatment of frozen shoulder. These could lead to better understanding of this common, protracted and painful condition.

In our study we found excellent results ( $88 \%$ average) with hydraulic distension technique. On the basis of the findings in our study we recommend that patients with frozen shoulder should be treated with hydraulic distension under local anesthesia with steroid because this technique is safe and cost effective and provide immediate relief of symptoms and early return to nearly full function.

Our patients in group-II, who were given only intra-articular steroid, gave fair to good results (70\% average) but most of them were not satisfied. Because the improvement in ROM of shoulder was much lesser in these patients $63 \%$ as compare to the patients in group-I (84\%) being treated by hydraulic distension technique.

\section{CONCLUSION}

We conclude that hydraulic distension technique under local anesthesia with steroid should be considered first in the management of frozen shoulder.

\section{REFERENCES}

1. Bunker TD, Reilly J, Baird KS, et al. Expression of growth factors, Cytokines and matrix metalloproteinases in frozen shoulder. $J$ Bone Joint surg. (Br) 2000; 82-B: 768-73.

2. Bunker TD and Anthony PP. The pathology of frozen shoulder a dupuytren like disease. J Bone joint surg (Br) 1995.77-B: 83.

3. Griggs SM, Anthony AHN, Green A, Idepatheic Adhesive capsulitis, $A$ prospective functional out come study or non operative treatment. JBJS (US). Incorporated 200; 10:13981207.

4. Ekalund $A L$ and Rydell $N$. Combination treatment of Adhesive capsulitis of the shoulder. Clin. Orthop; 282.1992; 105-109.

5. Shaffer B, Tibone JE, Kerlan RK. Frozen shoulder. A long term follow up J bone. Joint Surgery. (Am) 1992; 74-A: 738-746.

6. Murnaghan JP. Frozen shoulder in Rockwood CA. Matsen FA. 111 (eds). The shoulder Philadelphia, Pa W.B. Saunders 1990; 837-862.

7. Kuhn JE. Blasier R.B, Assessment of outcome of shoulder arthropalasty. Orthpaedic Clinic of North America 1998; 29(3): 549-563.

8. Constant C.R. Murley AHG. A clinical method of functional assessment of 
the shoulder. Clin Orhto 1997; 214: 160-164.

9. Ozaki J, Nakagawa Y, Sakural G and Tamia S. Recalcitrant Chronic Adhesive Capsulitis of the shoulder. Role of contracture of the corachumeral ligament and rotator interval in pathogenesis and treatment. J. Bone and Joint Surg 1989; 71-A: 1511-1515.

10. Rizk TE, Pinals RS, Talaiver AS. Corticosteroid injections in adhesive capsulitis. Investigation of their value and site. Arch, Phys. Med. Rehabil. 1991; 71 (1): 20-22.

11. Fareed Do and Gallivan WR Office management frozen shoulder syndrome. Treatment with hydraulic distention under local anesthesia. Clin. Ortho. 1989: 242: 177-183.

12. Gam AN. Schydlowsky $P$, Rossel IB, Remvig $L$, and Jensen $E M$, Treatment of frozen shoulder with distension ad Glucorticoid compared with glcorticoid alone. A randomized controlled trail. Scand. J. Rheumatol 1998; 27: 424-30.

13. Bland JH, Merrit JA, and Boushey DR. The Painful shoulder Semin. Arhritis Rehum 1977; (10: 21-47.

14. Sharma RK, Baje Kal RA, Bhan S. Frozen shoulder syndrome. A comparison of hydraulic distension and manipulation. Int. ortho 1993; 17(5): 275-8.

15. Van Royen BJ, Pavlov PW, Treatment of frozen shoulder by distension and manipulation under local anaesthesia. Int. Orthop. 1996: 20 (4); 207-10.

16. Wolf Jm.Green A. influence of comorbidity on self -assement instrument scores of patients with idiopathic adhesive capsulitis $J$ Bone joint surgAm, 2002:84:A:1167[(PMID) 12107317)] 